\title{
Self-dual interval orders and row-Fishburn matrices
}

\author{
Sherry H. F. Yan* \\ Department of Mathematics \\ Zhejiang Normal University \\ Jinhua 321004, P.R. China \\ huifangyan@hotmail.com
}

\author{
Yuexiao $\mathrm{Xu}$ \\ Department of Mathematics \\ Zhejiang Normal University \\ Jinhua 321004, P.R. China \\ xyx1985@163.com
}

Submitted: Nov 18, 2011; Accepted: Mar 29, 2012; Published: Apr 7, 2012

Mathematics Subject Classifications: 05A05, 05C30

\begin{abstract}
Recently, Jelínek derived that the number of self-dual interval orders of reduced size $n$ is twice the number of row-Fishburn matrices of size $n$ by using generating functions. In this paper, we present a bijective proof of this relation by establishing a bijection between two variations of upper-triangular matrices of nonnegative integers. Using the bijection, we provide a combinatorial proof of the refined relations between self-dual Fishburn matrices and row-Fishburn matrices in answer to a problem proposed by Jelínek.
\end{abstract}

Keywords: self-dual interval order; self-dual Fishburn matrix; row-Fishburn matrix.

\section{Introduction}

A poset is said to be an interval order (also known as $(2+2)$-free poset) if it does not contain an induced subposet that is isomorphic to $2+2$, the union of two disjoint 2element chains. Let $P$ be a poset with a strict order relation $\prec$. A strict down-set of an element $x \in P$ is the set $D(x)$ of all the elements of $\mathrm{P}$ that are smaller than $x$, i.e., $D(x)=\{y \in P: y \prec x\}$. Similarly, the strict up-set of $x$, denoted by $U(x)$, is the set $\{y \in P: y \succ x\}$. A poset $P$ is $(2+2)$-free if and only if its sets of strict down-sets, $D(P)=\{D(x): x \in P\}$ can be written as

$$
D(P)=\left\{D_{1}, D_{2}, \ldots, D_{m}\right\}
$$

*Supported by the National Natural Science Foundation of China (No. 10901141), Zhejiang Innovation Project(Grant No. T200905) and Opening Fund of Top Key Discipline of Computer Software and Theory in Zhejiang Provincial Colleges at Zhejiang Normal University. 
where $\emptyset=D_{1} \subset D_{2} \subset \ldots \subset D_{m}$, see $[1,2]$. In such context, we say that $x \in P$ has level $i$ if $D(x)=D_{i}$. An element has level 1 if and only if it is minimal. Following Fishburn [7], we call the number $m$ of distinct strict down-sets the magnitude of $\mathrm{P}$. It turns out that $m$ is also equal to the number of distinct strict up-sets, and we can order the strict up-sets of $P$ into a decreasing chain

$$
D(P)=\left\{U_{1}, U_{2}, \ldots, U_{m}\right\}
$$

where $U_{1} \supset U_{2} \supset \ldots \supset U_{m}=\emptyset$, see $[7,8]$. We say that $x$ has up-level $i$ if $U(x)=U_{i}$. An element has up-level $m$ if and only if it is maximal.

The dual of a poset $P$ is the poset $\bar{P}$ with the same elements as $P$ and an order relation $\prec$ defined by $x \prec y \Longleftrightarrow y \prec x$. A poset is self-dual if it is isomorphic to its dual.

Fishburn [7, 9] did pioneering work on interval orders; for instance, he provided several equivalent characterizations of interval orders and established a bijection between interval orders and a certain kind of integer matrices, called Fishburn matrices. Recently, Bousquet-Mélou et al. [2] constructed bijections between interval orders and ascent sequences, between ascent sequences and permutations avoiding a certain pattern, between interval orders and regular linearized chord diagrams by Stoimenow [12]. Several other papers have focused on bijections between interval orders and other objects. For instance, Dukes and Parviainen [4] have described a direct bijection between Fishburn matrices and ascent sequences, while the papers of Claesson et al. [3] and Dukes et al. [6] extend the bijection between interval orders and Fishburn matrices to more general combinatorial structures.

A Fishburn matrix of size $n$ is an upper-triangular matrix with nonnegative integers which sum to $n$ and each row and each column contains a nonzero entry. Throughout this paper we assume that each matrix has its rows numbered from top to bottom, and columns numbered left-to-right, starting with row and column number one. We let $M_{i, j}$ denote the entry of $\mathrm{M}$ in row $i$ and column $j$. The size of a matrix $M$ is the sum of all its entries. Moreover, the dimension of an upper triangular matrix is defined to the number of rows.

The dual matrix of $M$, denoted by $\bar{M}$, is obtained from $M$ by transposition along the diagonal running from bottom-left to top-right. More precisely, for $1 \leqslant i, j \leqslant m$, we have $\bar{M}_{i, j}=M_{m+1-j, m+1-i}$ where $m$ is the dimension of $M$. If a matrix $\mathrm{M}$ is equal to $\bar{M}$, we call it self-dual.

Fishburn [7, 9] showed that an interval order $P$ of magnitude $m$ corresponds to an $m \times m$ Fishburn matrix $M$ with $M_{i, j}$ being equal to the number of elements of $P$ that have level $i$ and up-level $j$. Jelínek [10] showed that the Fishburn's bijection turns out to be a bijection between self-dual interval orders of size $n$ and self-dual Fishburn matrices of size $n$.

Following the terminologies given in [10], we distinguish three types of cells in a Fishburn matrix $M$ of dimension $k$ : a cell $(i, j)$ is a diagonal cell if $i+j=k+1$, i.e., $(i, j)$ belongs to the north-east diagonal of the matrix. If $i+j<k+1$ (i.e., $(i, j)$ is above and to the left of the diagonal) then $(i, j)$ is a North-West cell, or NW-cell, while if $i+j>k+1$, then $(i, j)$ is an SE-cell. Clearly, NW-cells and diagonal cells together determine a self- 
dual Fishburn matrix. The reduced size of a self-dual fishburn matrix $M$ is the sum of all diagonal cells and NW-cells. The reduced size of a self-dual interval order $P$ is the reduced size of its corresponding self-dual Fishburn matrix under Fishburn's bijection.

A row-Fishburn matrix of size $n$ is defined to be an upper-triangular matrix with nonnegative integers which sum to $n$ and each row contains a nonzero entry. In a matrix $A$, the sum of a column (resp. row) is defined to the sum of all the entries in this column (resp. row). A column or a row is said to be zero if it contains no nonzero entries. The set of self-dual Fishburn matrices of reduced size $n$ is denoted by $\mathcal{M}(n)$. Let $\mathcal{M}(n, k, p)$ be the set of self-dual Fishburn matrices of reduced size $n$ whose first row has sum $k$ and diagonal cells have sum $p$. Let $\mathcal{R} \mathcal{M}(n)$ be the set of row-Fishburn matrices of size $n$. The set of row-Fishburn matrices in $\mathcal{R} \mathcal{M}(n)$ whose last column has sum $k$ is denoted by $\mathcal{R} \mathcal{M}(n, k)$. Let $\mathcal{R} \mathcal{M}(n, k, p)$ denote the set of row-Fishburn matrices in $\mathcal{R} \mathcal{M}(n, k)$ whose first row has sum $p$. Moreover, the set of self-dual interval orders of reduced size $n$ is denoted by $\mathcal{I}(n)$.

Based on the bijection between interval orders and Fishburn matrices, Jelínek [10] presented a new method to derive formulas for the generating functions of interval orders, counted with respect to their size, magnitude, and number of minimal and maximal elements, which generalize previous results on refined enumeration of interval orders obtained by Bousquet-Mélou et al. [2], Kitaev and Remmel [11], and Dukes et al. [5]. Applying the new method, Jelínek [10] obtained formulas for the generating functions of self-dual interval orders with respect to analogous statistics. From the obtained generating functions, relations between self-dual Fishburn matrices and row-Fishburn matrices were derived, that is,

$$
|\mathcal{M}(n, k, 0)|=|\mathcal{R} \mathcal{M}(n, k)|
$$

and for $p \geqslant 1$

$$
|\mathcal{M}(n, k, p)|=|\mathcal{R} \mathcal{M}(n, k, p)| .
$$

Combining the bijection between self-dual interval orders and self-dual Fishburn martices, formulas (1) and (2), Jelínek derived that for $n \geqslant 1$,

$$
|\mathcal{I}(n)|=|\mathcal{M}(n)|=2|\mathcal{R} \mathcal{M}(n)|
$$

and asked for bijective proofs of (1) and (2). The main objective of this paper is to present bijective proofs of these formulas by establishing a one-to-one correspondence between two variations of upper-triangular matrices of nonnegative integers.

Let $\mathcal{M}(n, k)$ be the set of self-dual Fishburn matrices of reduced size $n$ whose first row has sum $k$. Denote by $\mathcal{E} \mathcal{M}(n, k)$ (resp. $\mathcal{O} \mathcal{M}(n, k)$ ) the set of self-dual Fishburn matrices in $\mathcal{M}(n, k)$ whose dimension are even (resp. odd). Using the bijection between two variations of upper-triangular matrices of nonnegative integers, we derive that

$$
|\mathcal{E} \mathcal{M}(n, k)|=|\mathcal{O} \mathcal{M}(n, k)|=|\mathcal{R} \mathcal{M}(n, k)|
$$




\section{The bijective proofs}

Recall that a self-dual Fishburn matrix is determined by its NW-cells and diagonal cells. Given a self-dual Fishburn matrix $M$, the reduced matrix of $M$, denoted by $R(M)$, is a matrix obtained from $M$ by filling all the SE-cells with zeros. An upper-triangular matrix is said to be a super triangular matrix if all its SE-cells are zero.

Lemma 1. Let $M^{\prime}$ be a super triangular matrix of dimension $m$. Then $M^{\prime}$ is a reduced matrix of a self-dual Fishburn matrix if and only if it satisfies the following two conditions:

(i) for $1 \leqslant i \leqslant\left\lceil\frac{m}{2}\right\rceil$, each column $i$ contains a nonzero entry;

(ii) for $1 \leqslant i \leqslant\left\lceil\frac{m}{2}\right\rceil$, either row $i$ or column $m+1-i$ contains a nonzero entry.

Proof. Let $M$ be a self-dual Fishburn matrix with $R(M)=M^{\prime}$. Clearly, $M^{\prime}$ is a super triangular matrix. Since the first $\left\lceil\frac{m}{2}\right\rceil$ columns of $M^{\prime}$ are the same as those in $M$, the condition $(i)$ follows immediately. It remains to show that $M^{\prime}$ satisfies condition $(i i)$. Since $M$ is self-dual Fishburn matrix, for all $1 \leqslant i \leqslant m$, row $i$ must contains a nonzero entry, that is,

$$
\sum_{j=1}^{m} M_{i, j}=\sum_{j=1}^{m-i} M_{i, j}+\sum_{j=m+1-i}^{m} M_{i, j}=\sum_{j=1}^{m-i} M_{i, j}+\sum_{j=1}^{i} M_{j, m+1-i}>0 .
$$

Hence, for $1 \leqslant i \leqslant\left\lceil\frac{m}{2}\right\rceil$, either row $i$ or column $m+1-i$ of $R(M)$ contains a nonzero entry. Therefore, the condition ( $i i)$ holds for $R(M)$.

Conversely, given a super triangular matrix $M^{\prime}$ satisfying conditions $(i)$ and $(i i)$, We can recover a self-dual matrix $M$ from $M^{\prime}$ by filling the SE-cell $(m+1-j, m+1-i)$ with $M_{i, j}^{\prime}$. If $1 \leqslant i \leqslant\left\lceil\frac{m}{2}\right\rceil$, the sum of row $i$ of $M$ is given by

$$
\sum_{j=i}^{m} M_{i, j}=\sum_{j=i}^{m-i} M_{i, j}+\sum_{j=m+1-i}^{m} M_{i, j}=\sum_{j=1}^{m-i} M_{i, j}+\sum_{j=1}^{i} M_{j, m+1-i}=\sum_{j=1}^{m-i} M_{i, j}^{\prime}+\sum_{j=1}^{i} M_{j, m+1-i}^{\prime}
$$

By the condition $(i i)$, we have $\sum_{j=i}^{m} M_{i, j}=\sum_{j=1}^{m-i} M_{i, j}^{\prime}+\sum_{j=1}^{i} M_{j, m+1-i}^{\prime}>0$, which implies that row $i$ contains a nonzero entry. If $\left\lceil\frac{m}{2}\right\rceil+1 \leqslant i \leqslant m$, the sum of row $i$ of $M$ is given by

$$
\sum_{j=i}^{m} M_{i, j}=\sum_{j=i}^{m} M_{m+1-j, m+1-i}^{\prime}=\sum_{j=1}^{m+1-i} M_{j, m+1-i}^{\prime}
$$

which implies that the sum of row $i$ of $M$ is the same as that of column $m+1-i$ of $M^{\prime}$. By condition $(i)$, row $i$ contains a nonzero entry. Hence $M$ is a self-dual Fishburn matrix with $R(M)=M^{\prime}$. This completes the proof.

Denote by $\mathcal{S M}_{k}(n)$ the set of all super triangular matrices of size $n$ and dimension $2 k+1$ having the following two properties: 
(a) for $1 \leqslant i \leqslant k$, each column $i$ contains a nonzero entry;

(b) for $1 \leqslant i \leqslant k$, either row $k+1-i$ or column $k+1+i$ contains a nonzero entry.

Let $\mathcal{S} \mathcal{M}(n)=\bigcup_{k \geqslant 0} \mathcal{S M}_{k}(n)$.

Now we proceed to present a map $\alpha$ from $\mathcal{M}(n)$ to $\mathcal{S} \mathcal{M}(n)$. Given a nonempty selfdual matrix $M$ of dimension $m$, let $\alpha(M)$ be the matrix obtained from $M$ by the following procedure.

- If $m=2 k+1$ for some integer $k \geqslant 0$, then let $\alpha(M)$ be the matrix obtained from the reduced matrix $R(M)$ of $M$ by interchanging the cell $(i, k+1)$ and the diagonal cell $(i, m+1-i)$ for $1 \leqslant i \leqslant k$.

- If $m=2 k$ for some integer $k \geqslant 1$, then let $A$ be the matrix obtained from $R(M)$ by adding one zero row and one zero column immediately after column $k$ and row $k$. Define $\alpha(M)$ to be the matrix obtained from $A$ by interchanging the cell $(i, k+1)$ and the diagonal cell $(i, m+1-i)$ of the resulting matrix $A$.

Obviously, $\alpha(M)$ is a super triangular matrix of dimension $2 k+1$ and size $n$. It easy to check that the map $\alpha$ preserves the first $k$ columns and the total sum of row $i$ and column $m+1-i$ of the reduced matrix $R(M)$. By Lemma 1, the matrix $\alpha(M)$ has properties (a) and $(b)$. Hence $\alpha(M)$ is a super triangular matrix in $\mathcal{S} \mathcal{M}(n)$.

Conversely, given a super triangular matrix $M^{\prime}$ in $\mathcal{S} \mathcal{M}(n)$ of dimension $2 k+1$, we can recover a matrix $M \in \mathcal{M}(n)$ with $\alpha(M)=M^{\prime}$. First we interchange the cell $(i, k+1)$ with the diagonal cell $(i, m-i)$ for $1 \leqslant i \leqslant k$. Then we obtain a matrix $A$ by deleting column $k+1$ and row $k+1$ if they are zero. It is easy to check that properties $(a)$ and $(b)$ ensure that the obtained matrix $A$ is the reduced matrix of a self-dual Fishburn matrix. Let $M$ be a self-dual Fishburn matrix with $R(M)=A$. Hence $\alpha$ is a bijection between $\mathcal{M}(n)$ and $\mathcal{S} \mathcal{M}(n)$.

Let $M$ be a super triangular matrix of dimension $2 k+1$, then column $k+1$ is called a center column. From the construction of the bijection $\alpha$, we see that the map $\alpha$ transforms the sum of the diagonal cells of a self-dual matrix to the sum of the center column of a super triangular matrix. Hence, we have the following result.

Theorem 2. The map $\alpha$ is a bijection between $\mathcal{M}(n)$ and $\mathcal{S} \mathcal{M}(n)$. Moreover, the bijection $\alpha$ preserves the sum of the first row, and transforms the sum of the diagonal cells of a self-dual matrix to the sum of the center column of a super triangular matrix.

Example 3. Consider a matrix $A \in \mathcal{M}(5)$,

$$
A=\left[\begin{array}{lllll}
1 & 0 & 1 & 0 & 0 \\
0 & 1 & 1 & 1 & 0 \\
0 & 0 & 0 & 1 & 1 \\
0 & 0 & 0 & 1 & 0 \\
0 & 0 & 0 & 0 & 1
\end{array}\right]
$$


The reduced matrix of $A$ is given by

$$
R(A)=\left[\begin{array}{lllll}
1 & 0 & 1 & 0 & 0 \\
0 & 1 & 1 & 1 & 0 \\
0 & 0 & 0 & 0 & 0 \\
0 & 0 & 0 & 0 & 0 \\
0 & 0 & 0 & 0 & 0
\end{array}\right],
$$

and we have

$$
\alpha(A)=\left[\begin{array}{lllll}
1 & 0 & 0 & 0 & 1 \\
0 & 1 & 1 & 1 & 0 \\
0 & 0 & 0 & 0 & 0 \\
0 & 0 & 0 & 0 & 0 \\
0 & 0 & 0 & 0 & 0
\end{array}\right] .
$$

Let $\mathcal{B}(n)$ the set of upper-triangular matrices of size $n$ in which each row contains a nonzero entry except for the first row. Given a nonempty matrix $A \in \mathcal{R} \mathcal{M}(n)$, we can get two distinct matrices in $\mathcal{B}(n)$ from $A$ by either doing nothing or adding a zero row and a zero column before the first row and the first column. Thus for $n \geqslant 1$ we have the following relation

$$
|\mathcal{B}(n)|=2|\mathcal{R} \mathcal{M}(n)| .
$$

Now we proceed to construct a bijection between the set $\mathcal{S} \mathcal{M}(n)$ and the set $\mathcal{B}(n)$. Before constructing the bijection, we need some definitions. In a matrix $A$ with $m$ rows, the operation of adding column $i$ to column $j$ is defined by increasing $A_{k, j}$ by $A_{k, i}$ for each $k=1,2, \ldots, m$.

Let $\mathcal{B}(n, k, p)$ be the set of matrices in $\mathcal{B}(n)$ whose whose first row has sum $p$ and last column has sum $k$. Similarly, let $\mathcal{S M}(n, k, p)$ be the set of matrices in $\mathcal{S M}(n)$ whose first row has sum $k$ and center column has sum $p$.

Theorem 4. For $n \geqslant 1$, there is a bijection $\beta$ between $\mathcal{S M}(n)$ and $\mathcal{B}(n)$. Moreover, the map $\beta$ is essentially a bijection between $\mathcal{S} \mathcal{M}(n, k, p)$ and $\mathcal{B}(n, k, p)$.

Proof. Given a nonempty triangular matrix $A \in \mathcal{S} \mathcal{M}(n)$ of dimension $2 k+1$, we recursively construct a sequence of super triangular matrices $A^{(0)}, A^{(1)}, \ldots, A^{(l)}$. Let $A^{(0)}=A$ and assume that we have obtained the matrix $A^{(j)}$. Let $A^{(j)}$ be a super triangular matrix of dimension $2 r+1$ for some integer $r$. For $1 \leqslant i \leqslant r$, if each column $r+1+i$ is zero, then let $A^{(l)}=A^{(j)}$. Otherwise, we proceed to generate the matrix $A^{(j+1)}$ by the following insertion algorithm.

- Find the largest value $i$ such that column $r+1+i$ contains a nonzero entry. Then fill the entries of column $r+1+i$ with zeros.

- Insert one column immediately after column $r+1-i$, one zero row immediately after row $r+1-i$, one zero column immediately before column $r+1+i$ and one 
zero row immediately before row $r+1+i$. Let the entry in row $j$ of the new inserted column after column $r+1-i$ be filled with the entry in row $j$ of column $r+1+i$ of $A^{(j)}$ for $1 \leqslant j \leqslant 2 r+1$.

Suppose that $A^{(l)}$ is of dimension $2 q+1$. Then the last $q$ rows and $q$ columns of $A^{(l)}$ are zero rows and columns. Let $B$ be an upper-triangular matrix obtained from $A^{(l)}$ by deleting the last $q$ columns and $q$ rows. From the above insertion procedure to generate $A^{(j+1)}$ form $A^{(j)}$, we see that the inserted column after column $r+1-i$ contains a nonzero entry. This ensures that each matrix $A^{(j)}$ has property $(a)$ with $0 \leqslant j \leqslant l$. Hence each column $i$ of $A^{(l)}$ contains a nonzero entry with $1 \leqslant i \leqslant q$. Hence, $B$ is an upper-triangular matrix in which each column contains a nonzero entry except for the last column. Moreover, the insertion algorithm preserves the sum of each nonzero row of $A$, which implies that $B$ is of size $n$. Let $\beta(A)$ be the dual matrix of $B$. Hence we have $\beta(A) \in \mathcal{B}(n)$.

Conversely, we can construct a matrix $A=\beta^{\prime}\left(A^{\prime}\right)$ in $\mathcal{S M}(n)$ from a matrix $A^{\prime}$ of dimension $k+1$ in $\mathcal{B}(n)$. Let $B$ be the dual matrix of $A^{\prime}$. Define $M$ to be a matrix of dimension $2 k+1$ obtained from $B$ by adding $k$ consecutive zero rows and $k$ consecutive zero columns immediately after column $k+1$ and row $k+1$. Clearly, the obtained matrix is a super triangular matrix having property $(a)$. If for all $1 \leqslant i \leqslant k$, either row $k+1-i$ or column $k+1+i$ contains a nonzero entry, then we do nothing for $M$ and let $A=M$. Otherwise, we can construct a new super triangular matrix $A$ by the following removal algorithm.

- Find the least value $i$ such that neither row $k+1-i$ nor column $k+1+i$ contains a nonzero entry. Then we obtain a super triangular matrix by adding column $k+1-i$ to column $k+2+i$ and removing columns $k+1+i, k+1-i$ and rows $k+1-i$, $k+1+i$.

- Repeat the above procedure for the resulting matrix until the obtained matrix has property $(b)$.

Obviously, the obtained matrix $A$ is a super triangular matrix having properties $(a)$ and (b). Since the algorithm preserves the sums of entries in each non-zero row of $B$, the matrix $A$ is of size $n$ and the sum of the first row of $A$ is the same as that of $B$. The property $(b)$ ensures that the inserted columns in the insertion algorithm are the removed columns in the removal algorithm. Thus the map $\beta^{\prime}$ is the inverse of the map $\beta$. From the construction of the removal algorithm, the sum of the center column of $A$ is equal to the sum of the last column of $B$ as well as the the sum of the first row of $A^{\prime}$. This completes the proof.

Example 5. Consider a matrix $A \in \mathcal{S M}(6)$,

$$
A=\left[\begin{array}{lllll}
1 & 0 & 0 & 1 & 1 \\
0 & 1 & 1 & 1 & 0 \\
0 & 0 & 0 & 0 & 0 \\
0 & 0 & 0 & 0 & 0 \\
0 & 0 & 0 & 0 & 0
\end{array}\right]
$$


Let $A^{(0)}=A$. By applying the insertion algorithm, we get

$$
\begin{gathered}
A^{(1)}=\left[\begin{array}{lllllll}
1 & \mathbf{1} & 0 & 0 & 1 & \mathbf{0} & 0 \\
\mathbf{0} & \mathbf{0} & \mathbf{0} & \mathbf{0} & \mathbf{0} & \mathbf{0} & \mathbf{0} \\
0 & \mathbf{0} & 1 & 1 & 1 & \mathbf{0} & 0 \\
0 & \mathbf{0} & 0 & 0 & 0 & \mathbf{0} & 0 \\
0 & \mathbf{0} & 0 & 0 & 0 & \mathbf{0} & 0 \\
\mathbf{0} & \mathbf{0} & \mathbf{0} & \mathbf{0} & \mathbf{0} & \mathbf{0} & \mathbf{0} \\
0 & \mathbf{0} & 0 & 0 & 0 & \mathbf{0} & 0
\end{array}\right], \\
A^{(2)}=\left[\begin{array}{lllllllll}
1 & 1 & 0 & \mathbf{1} & 0 & \mathbf{0} & 0 & 0 & 0 \\
0 & 0 & 0 & \mathbf{0} & 0 & \mathbf{0} & 0 & 0 & 0 \\
0 & 0 & 1 & \mathbf{1} & 1 & \mathbf{0} & 0 & 0 & 0 \\
\mathbf{0} & \mathbf{0} & \mathbf{0} & \mathbf{0} & \mathbf{0} & \mathbf{0} & \mathbf{0} & \mathbf{0} & \mathbf{0} \\
0 & 0 & 0 & \mathbf{0} & 0 & \mathbf{0} & 0 & 0 & 0 \\
\mathbf{0} & \mathbf{0} & \mathbf{0} & \mathbf{0} & \mathbf{0} & \mathbf{0} & \mathbf{0} & \mathbf{0} & \mathbf{0} \\
0 & 0 & 0 & \mathbf{0} & 0 & \mathbf{0} & 0 & 0 & 0 \\
0 & 0 & 0 & \mathbf{0} & 0 & \mathbf{0} & 0 & 0 & 0 \\
0 & 0 & 0 & \mathbf{0} & 0 & \mathbf{0} & 0 & 0 & 0
\end{array}\right],
\end{gathered}
$$

where the inserted rows and columns are illustrated in bold at each step of the insertion algorithm. Removing the last 4 zero rows and 4 zero columns, we get

$$
B=\left[\begin{array}{lllll}
1 & 1 & 0 & 1 & 0 \\
0 & 0 & 0 & 0 & 0 \\
0 & 0 & 1 & 1 & 1 \\
0 & 0 & 0 & 0 & 0 \\
0 & 0 & 0 & 0 & 0
\end{array}\right] .
$$

Finally, we obtain

$$
A^{\prime}=\beta(A)=\left[\begin{array}{lllll}
0 & 0 & 1 & 0 & 0 \\
0 & 0 & 1 & 0 & 1 \\
0 & 0 & 1 & 0 & 0 \\
0 & 0 & 0 & 0 & 1 \\
0 & 0 & 0 & 0 & 1
\end{array}\right]
$$

Conversely, given $A^{\prime} \in \mathcal{B}(6)$, by applying removal algorithm, we can get $A \in \mathcal{S} \mathcal{M}(6)$, where the removed rows and columns are illustrated in bold at each step of the removal algorithm.

Combining the bijection between self-dual interval orders and self-dual Fishburn matrices and Theorems 2 and 4 , we get a bijective proof of (3).

From Theorems 2 and 4, we have

$$
|\mathcal{M}(n, k, p)|=|\mathcal{S} \mathcal{M}(n, k, p)|=|\mathcal{B}(n, k, p)| .
$$

Given a matrix $M \in \mathcal{B}(n, k, 0)$, we can get a matrix $A \in \mathcal{R} \mathcal{M}(n, k)$ by deleting the first row and the first column. Conversely, given a matrix $A^{\prime} \in \mathcal{R} \mathcal{M}(n, k)$, we can obtain a 
matrix $M^{\prime} \in \mathcal{B}(n, k, 0)$ by inserting a zero row and a zero column before the first row and the first column. This yields that

$$
|\mathcal{M}(n, k, 0)|=|\mathcal{B}(n, k, 0)|=|\mathcal{R} \mathcal{M}(n, k)|
$$

If $p>0$, then $\mathcal{B}(n, k, p)$ is the same as $\mathcal{R} \mathcal{M}(n, k, p)$. Hence, if $p>0$ then we have

$$
|\mathcal{M}(n, k, p)|=|\mathcal{B}(n, k, p)|=|\mathcal{R} \mathcal{M}(n, k, p)|
$$

Therefore, we get combinatorial proofs of (1) and (2), in answer to the problem posed by Jelínek [10].

Now we proceed to prove (4). Given a matrix $A \in \mathcal{E} \mathcal{M}(n, k)$ of dimension $2 m$ for some integers $m \geqslant 1$, let $R(A)$ be its reduced matrix. We obtain a super triangular matrix $A^{\prime}$ from $A$ by inserting a zero column and a zero row immediately after column $m$ and row $m$. By Lemma 1 , we have $A^{\prime} \in \mathcal{S} \mathcal{M}(n, k, 0)$.

Conversely, given a matrix $A^{\prime} \in \mathcal{S} \mathcal{M}(n, k, 0)$ of dimension $2 m+1$ for some integer $m \geqslant 1$, we can recover a self-dual matrix $A \in \mathcal{E} \mathcal{M}(n, k)$ as follows. First, we get a super triangular matrix $B$ from $A^{\prime}$ by deleting column $m+1$ and row $m+1$. Let $A$ be a matrix with $B=R(A)$. Obviously, we have the matrix $A \in \mathcal{E} \mathcal{M}(n, k)$. Hence, we get that $|\mathcal{E} \mathcal{M}(n, k)|=|\mathcal{S} \mathcal{M}(n, k, 0)|$. By $(6)$, we deduce that

$$
|\mathcal{E} \mathcal{M}(n, k)|=|\mathcal{S} \mathcal{M}(n, k, 0)|=|\mathcal{R} \mathcal{M}(n, k)|
$$

From (6) and (7), we obtain that

$$
\begin{aligned}
|\mathcal{M}(n, k)| & =|\mathcal{M}(n, k, 0)|+\sum_{p \geqslant 1}|\mathcal{M}(n, k, p)| \\
& =|\mathcal{R} \mathcal{M}(n, k)|+\sum_{p \geqslant 1}|\mathcal{R} \mathcal{M}(n, k, p)| \\
& =2|\mathcal{R} \mathcal{M}(n, k)| .
\end{aligned}
$$

Meanwhile, we have $|\mathcal{M}(n, k)|=|\mathcal{E} \mathcal{M}(n, k)|+|\mathcal{O} \mathcal{M}(n, k)|$. Hence, (4) follows from (8).

\section{Acknowledgements}

The authors would like to thank the referee for helpful suggestions.

\section{References}

[1] K.P. Bogart. An obvious proof of Fishburn's interval order theorem. Discrete Math., 118:239-242, 1993.

[2] M. Bousquet-Mélou, A. Claesson, M. Dukes, and S. Kitaev. (2+2)-free posets, ascent sequences and pattern avoiding permutations. J. Combin. Theory Ser. A, 117:884-909, 2010.

[3] A. Claesson, M. Dukes, and M. Kubitzke. Partition and composition matrices. J. Combin. Theory Ser. A, 118:1624-1637, 2011. 
[4] M. Dukes, R. Parviainen. Ascent sequences and upper triangular matrices containing non-negative integers. Electronic J. Combin., 17:R53, 2010.

[5] M. Dukes, S. Kitaev, J. Remmel, and E. Steingrímsson. Enumerating (2+2)-free posets by indistinguishable elements. Journal of Combinatroics, 2:139-163, 2011.

[6] M. Dukes, V. Jelínek, and M. Kubitzke. Composition matrices, (2+2)-free posets and their specializations. Electronic J. Combin., 18:P44, 2011.

[7] P. C. Fishburn. Interval lengths for interval orders: A minimization problem. Discrete Math., 47:63-82, 1983.

[8] P. C. Fishburn. Interval graphs and interval orders. Discrete Math., 55:135-149, 1985.

[9] P. C. Fishburn. Interval orders and interval graphs: A study of partially ordered sets. John Wiley \& Sons, 1985.

[10] V. Jelínek. Counting general and self-dual interval orders. J. Combin. Theory Ser. A, 119:599-614, 2012.

[11] S. Kitaev, J. Remmel. Enumerating $(2+2)$-free posets by the number of minimal elements and other statistics. Discrete Appl. Math., 159:2098-2108, 2011.

[12] A. Stoimenow. Enumumeration of chord diagrams and an upper bound for Vassiliev invariants. J. Knot Theory Ramifications, 7:93-114, 1998.

[13] D. Zagier. Vassiliev invariants and a strange identity related to the Dedekind etafunction. Topology, 40:945-960, 2001. 